\title{
Research on Construction Method of Urban Driving Cycle of Pure Electric Vehicle
}

\author{
Xiao $\mathrm{Li}^{12^{2 *}}$, Zhifei Pang ${ }^{1}$, and Hongxue Zhao ${ }^{1}$ \\ ${ }^{1}$ Research Institute of Highway Ministry of Transport, Beijing 100088, China \\ ${ }^{2}$ School of Mechanical Engineering, Beijing Institute of Technology, Beijing 100081, China
}

\begin{abstract}
The driving cycle of the vehicle is taken as the basis of the vehicle test, which plays an important role in improving vehicle performance and reducing energy consumption. Traditional fuel vehicles have been studied more in the current stage. Test conditions specifically for pure electric vehicles have been less studied. The data acquisition method of pure electric vehicle is studied and used to collect driving data. The driving cycle was established through the extraction and analysis of characteristic parameters. The research results can lay a foundation for the research of driving system optimization and energy consumption reduction of pure electric vehicles.
\end{abstract}

\section{Introduction}

With the aggravation of energy shortage and environmental pollution, electric vehicles have been vigorously promoted as an important means to reduce energy consumption and environmental pollution. Electric vehicles have been vigorously developed along with important significance such as energy security guarantee, automobile industry progress in China.

Driving cycle is a common core technology in automobile industry. It is used to determine vehicle pollutant emissions, fuel consumption, technology development assessments for new models, and traffic control risk testing. The final result will be greatly affected by whether the determination of vehicle driving conditions is scientific. The determination of vehicle running cycle has been widely concerned by scholars. Millo F used NEDC and WLTP conditions as test cycles when designing and developing PHEV light commercial vehicles ${ }^{[1]}$. Amirjamshidi $G$ and Roorda $M J$ took the Toronto coastal area as an example, simulated the data of light, medium and heavy trucks in the morning traffic rush hour, adopted multi-objective genetic algorithm to calibrate the micro traffic simulation model, and developed a set of simulated driving cycles ${ }^{[2]}$. Fotouhi A and Montazeri-Gh $\mathrm{M}$ installed advanced vehicle positioning equipment on private cars, real vehicle driving data was collected, and a set of driving cycle in line with the actual conditions was calculated and constructed ${ }^{[3]}$. Liu $\mathrm{Z}$ et al. carried out research on the operation conditions of pickup trucks, and a method of synthesis from natural driving data was proposed ${ }^{[4]}$. Liu $\mathrm{D}$ et al. took HEV as the research object and built the transfer probability matrix of demand power under the condition of known driving conditions. Furthermore, the energy management strategy of HEV was studied ${ }^{[5]}$.

In the above studies, more attention has been paid to conventional fuel vehicles. Compared with fuel vehicles, electric vehicles have great differences in energy source, power transmission, driving range and other aspects. The EV driving cycle has been less studied at the current stage. However, the determination of the EV driving cycle plays an important role. Scientific driving cycle can lay an important foundation for optimization of driving system design, reduction of energy consumption and increase of driving range. Therefore, it is necessary to study the driving cycle of pure electric vehicles.

\section{Driving characteristic data acquisition}

In China, the urban road traffic structure is complex, the situation of non-mixed traffic is frequent, and the vehicle operating area changes greatly. Therefore, it is impossible to carry out the acquisition of vehicle operating data in a comprehensive way. In this paper, Shenzhen, the city with the largest number of pure electric vehicles and the strongest representative operating characteristics, is selected as the sample city, and the driving data of vehicles with regular driving in a period of time is selected for research.

In terms of operation data collection, according to the operation characteristics of pure electric vehicles, the test method of "real vehicle follow-up" is proposed, which is based on the average traffic flow method, vehicle tracking method and autonomous driving method that are commonly used at present. The implementation steps of this method are as follows:

(1) The representative sample vehicles were analyzed and selected.

\footnotetext{
* Corresponding author: x.li@rioh.cn
} 
(2) Data acquisition instruments are installed on allelectric vehicles.

(3) The experimental personnel accompany the vehicle to collect vehicle operation data.

(4) The data collected by equipment and the data collected by personnel are summarized and compared to form the final database.

On the one hand, the collected data can be guaranteed to be real, and there is a strong scientific nature through this method. On the other hand, the normal running of the vehicle is not affected, and the degree of cooperation is high

There are many driving parameters of pure electric vehicles in operation. The purpose of this collection is to measure and record the velocity - time curve and the corresponding velocity and acceleration distribution characteristics of the bus in actual operation. Therefore, the real-time data of bus travelling speed, time and mileage are mainly collected in the test.

The acceleration of vehicle was used for subsequent data analysis. But the acceleration signal was not directly collected in the test. Therefore, the acceleration at each time point must be calculated. The calculation formula of acceleration is as follows:

$$
a_{i}=\frac{V_{i+1}-V_{i}}{t_{i+1}-t_{i}}, i=1,2, \cdots, k-1
$$

Where $a_{i}$ is the acceleration of the sampling point $i$, $V_{i}, V_{i+1}$ are the speed of sampling point $i$ and $i+1$, respectively; $t_{i}, t_{i+1}$ are the time of sampling point $i$ and $i+1$, respectively; $k$ is the number of sampling point.

\section{Driving characteristic parameter}

In order to describe the operating characteristics of operating conditions, some criterion parameters representing its motion characteristics need to be extracted. Mathematical basis can be provided for driving cycle analysis through these parameters. Nineteen characteristic parameters were selected to comprehensively describe the motion characteristics of short stroke, such as speed, acceleration, four operating states including acceleration, deceleration, constant speed, idle speed, the proportion of each speed interval.

The symbols and meanings of the 19 parameters are shown in Table 1.

Table 1. The symbols and meanings of the parameters

\begin{tabular}{|c|c|c|c|c|c|c|c|}
\hline Number & symbol & meaning & unit & Number & symbol & meaning & unit \\
\hline 1 & $V_{m}$ & Average velocity & $\mathrm{km} / \mathrm{h}$ & 11 & $P_{i}$ & Idle speed ratio & $\%$ \\
\hline 2 & $V_{\max }$ & Maximum speed & $\mathrm{km} / \mathrm{h}$ & 12 & $a_{m}$ & Mean acceleration & $\mathrm{m} / \mathrm{s}^{2}$ \\
\hline 3 & $V_{s d}$ & Speed standard deviation & - & 13 & $d_{m}$ & Mean deceleration & $\mathrm{m} / \mathrm{s}^{2}$ \\
\hline 4 & $a_{\max }$ & Maximum acceleration & $\mathrm{m} / \mathrm{s}^{2}$ & 14 & $P_{1}$ & Speed ratio in $0 \sim 10 \mathrm{~km} / \mathrm{h}$ & $\%$ \\
\hline 5 & $a_{\text {min }}$ & Minimum acceleration & $\mathrm{m} / \mathrm{s}^{2}$ & 15 & $P_{2}$ & Speed ratio in $10 \sim 20 \mathrm{~km} / \mathrm{h}$ & $\%$ \\
\hline 6 & $V_{m r}$ & Average speed & $\mathrm{km} / \mathrm{h}$ & 16 & $P_{3}$ & Speed ratio in $20 \sim 30 \mathrm{~km} / \mathrm{h}$ & $\%$ \\
\hline 7 & $a_{s d}$ & Acceleration standard deviation & - & 17 & $P_{4}$ & Speed ratio in $30 \sim 40 \mathrm{~km} / \mathrm{h}$ & $\%$ \\
\hline 8 & $P_{a}$ & Acceleration ratio & $\%$ & 18 & $P_{5}$ & Speed ratio in $40 \sim 50 \mathrm{~km} / \mathrm{h}$ & $\%$ \\
\hline 9 & $P_{d}$ & Reduction ratio & $\%$ & 19 & $P_{6}$ & Speed ratio above $50 \mathrm{~km} / \mathrm{h}$ & $\%$ \\
\hline 10 & $P_{c}$ & Constant speed ratio & $\%$ & -- & -- & -- & -- \\
\hline
\end{tabular}

In the process of data processing, the four running states are processed according to the following rules:

(1) Speed up: the continuous driving process when the acceleration value of the vehicle is greater than or equal to $0.15 \mathrm{~m} / \mathrm{s}^{2}$.

(2) Speed down: the continuous driving process when the acceleration value of the vehicle is less than or equal to $-0.15 \mathrm{~m} / \mathrm{s}^{2}$;

(3) Constant speed: the continuous driving process in which the acceleration value of the vehicle is between $\pm 0.15 \mathrm{~m} / \mathrm{s}^{2}$ and the speed is not 0 .

(4) Idle speed: The vehicle speed is 0 , and the acceleration is between plus and minus $0.15 \mathrm{~m} / \mathrm{s}^{2}$.

The calculation formula of some key parameters is as follows:

(1) Characteristic parameters of $V_{m}, V_{\max }, V_{s d}, V_{m r}$

$$
\begin{gathered}
V_{m}=\frac{\sum v(t), t=1,2, \cdots, T}{T} \\
V_{\max }=\max \{v(t), t=1,2, \cdots T\} \\
V_{m r}=\frac{\sum v(t), t=1,2, \cdots, T}{T-T_{i}}
\end{gathered}
$$

$$
V_{s d}=\sqrt{\frac{1}{T-1} \sum\left(v(t)-V_{m}\right)^{2}}, t=1,2, \cdots T
$$

(2) Characteristic parameters of $a_{m}, a_{\max } 、 a_{\min }, a_{s d}$, $a_{m}, d_{m}$

$$
\begin{gathered}
a_{m}=\frac{\sum a(t), t=1,2, \cdots, T}{T} \\
a_{\max }=\max \{a(t), t=1,2, \cdots T\} \\
a_{\min }=\min \{a(t), t=1,2, \cdots T\} \\
a_{s d}=\sqrt{\frac{1}{T-1} \sum(a(t))^{2}}, t=1,2, \cdots T \\
a_{m}=\frac{\sum a(t) \geq 0.15, t=1,2, \cdots, T}{T} \\
d_{m}=\frac{\sum a(t) \leq-0.15, t=1,2, \cdots, T}{T}
\end{gathered}
$$

\section{Construction of Driving Cycle}

According to the above research, the driving data of some pure electric vehicles driving regularly in Shenzhen 
during a period of time are collected, and the specific distribution is shown in Figure 1.

Before establishing the driving cycle, it is necessary to determine the structural parameters of the cycle based on the actual running characteristics of electric vehicles, which directly determine the shape and direction of the cycle diagram.

(1) The speed parameter

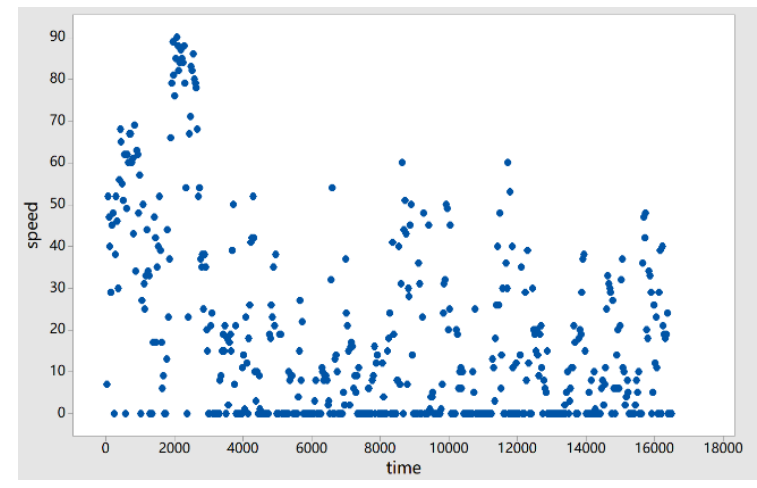

Fig. 1. Location time and speed distribution diagram
In terms of speed, average velocity, average speed, and maximum speed need to be calculated. The variation track of driving speed is shown in Figure 2. The cumulative probability distribution of velocity is shown in Figure 3.

As can be seen from Figure 2, the driving speed of pure electric vehicles varies frequently. As can be seen from Figure 3, the average velocity is $17.13 \mathrm{~km} / \mathrm{h}$. The average speed is $27.87 \mathrm{~km} / \mathrm{h}$. The maximum speed is $90 \mathrm{~km} / \mathrm{h}$. The minimum speed is $0 \mathrm{~km} / \mathrm{h}$.

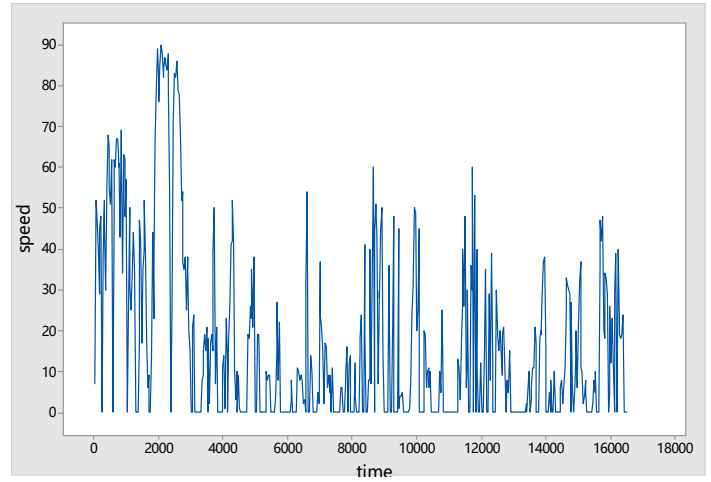

Fig. 2. The variation track of driving speed

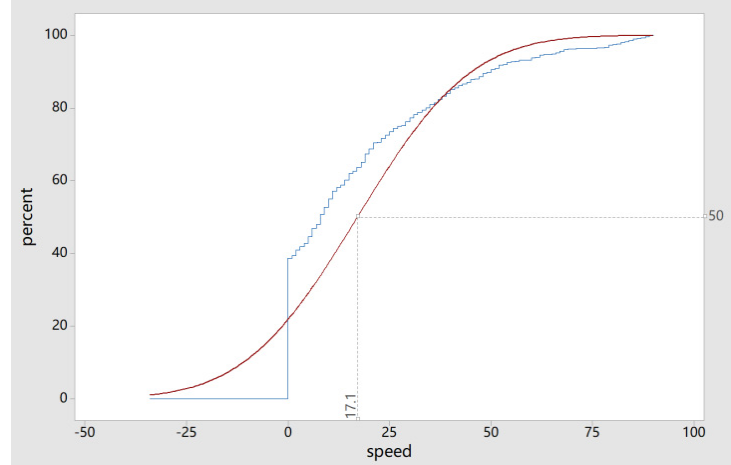

Fig. 3. Cumulative probability distribution of velocity

(2) The acceleration parameter

In terms of acceleration, maximum acceleration, maximum deceleration, average acceleration, average deceleration need to be calculated. The variation track of driving acceleration is shown in Figure 4.

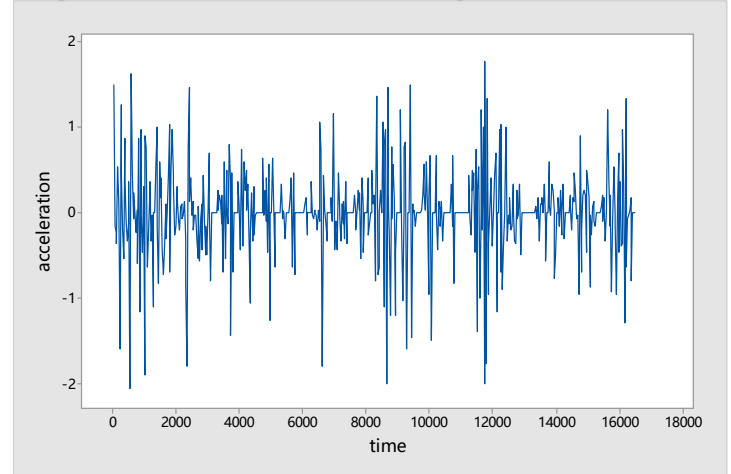

Fig. 4. The variation track of driving acceleration

As can be seen from Figure 4, the maximum acceleration is $1.77 \mathrm{~m} / \mathrm{s}^{2}$, the maximum deceleration is -
$2.07 \mathrm{~m} / \mathrm{s}^{2}$, the average acceleration is $0.55 \mathrm{~m} / \mathrm{s}^{2}$, the average deceleration is $-0.62 \mathrm{~m} / \mathrm{s}^{2}$.

(3) Ratio of travel state

In terms of travel state, the ratio of each travel state including acceleration, deceleration, constant speed and idle speed need to be calculated. The grouping of different driving speeds is shown in Fig. 5 After carrying out statistics and calculation on the data, the ratio of each travel state is shown in Figure 6.

As can be seen from Figure 5 and Figure 6, the ratio of acceleration is $34 \%$, the ratio of deceleration is $30 \%$, and the ratio of idle speed is $12 \%$. The ratio of $10 \mathrm{~km} / \mathrm{h}$ constant is $11 \%$, The ratio of $20 \mathrm{~km} / \mathrm{h}$ constant is $5 \%$, The ratio of $30 \mathrm{~km} / \mathrm{h}$ constant is $2 \%$, The ratio of $40 \mathrm{~km} / \mathrm{h}$ constant is $2 \%$, The ratio of $50 \mathrm{~km} / \mathrm{h}$ constant is $4 \%$. 


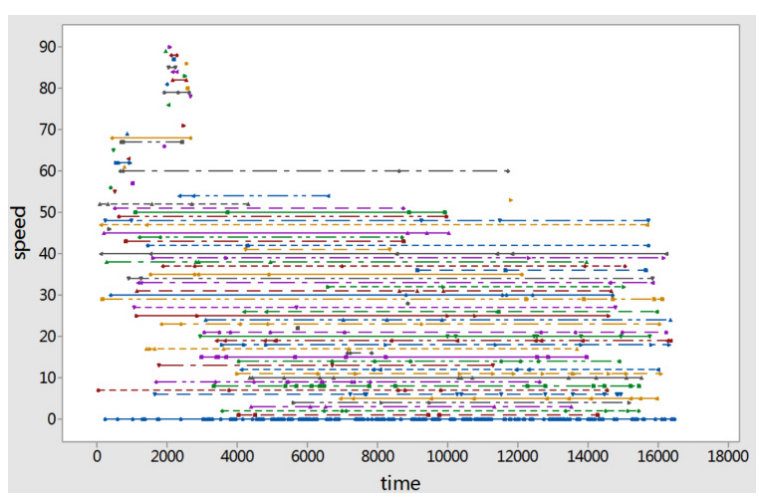

Fig. 5. The grouping of different driving speeds

Based on the operating characteristics and statistical analysis of pure electric vehicles, the corresponding characteristic parameters can be obtained, and then the operating cycle curve can be determined. The specific methods are as follows:

(1) According to the proportion of characteristic parameters of driving state, the proportion of acceleration, deceleration, uniform speed and idle speed in the cycle curve of test condition was obtained, and the length of each broken line in the cycle curve was determined according to the proportion.

(2) According to the characteristic parameter values of acceleration, the geometric features of acceleration and deceleration segments in the cycle curve of test conditions are determined. The maximum value of acceleration determines the maximum slope of the broken line in the rising segment, and the average value of acceleration determines the gentle condition of the rising segment in the overall curve. Similarly, the value of deceleration determines the slope of the descending polyline in the cycle curve.

(3) The height of the curve and the position of the broken line are determined according to the velocity characteristic value.

(4) The above broken lines are scientifically arranged to form the driving cycle curve.

Based on the above analysis, the driving cycle curve of electric vehicles is constructed, as shown in Fig. 7.

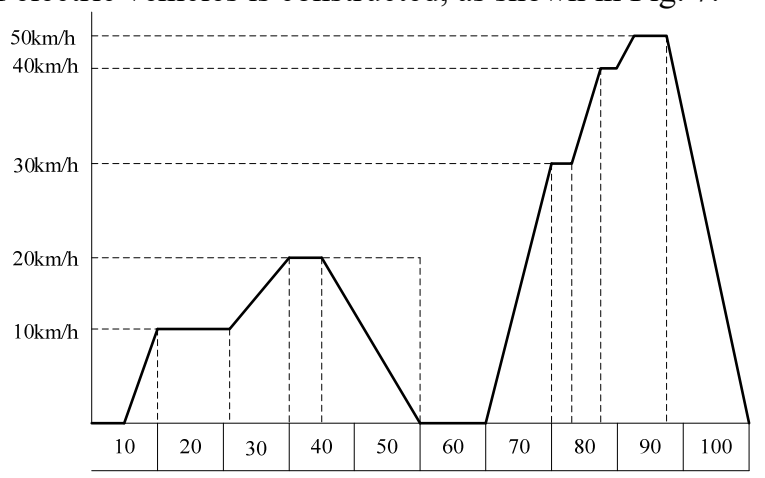

Fig. 7. The driving cycle curve of electric vehicles

\section{Conclusions}

In this paper, the driving cycle of pure electric vehicles is deeply analysed. The analysed data are collected during

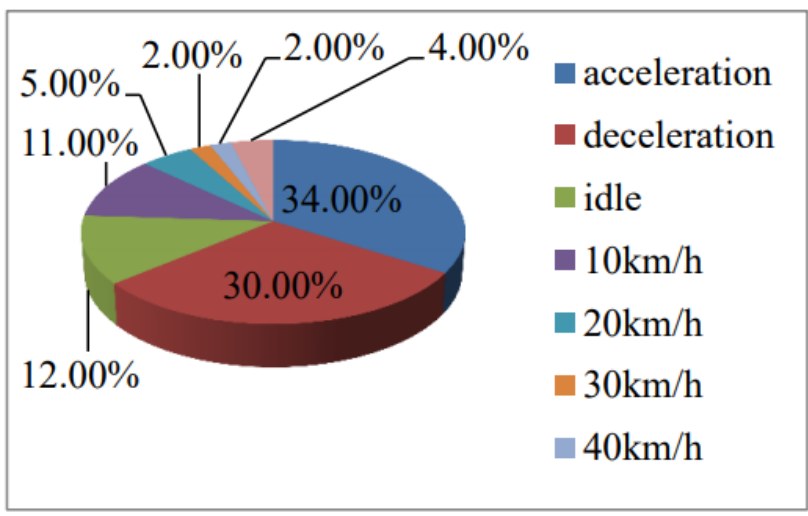

Fig. 6. The ratio of each travel state the actual operation. The research results can lay a foundation for the research of driving system optimization and energy consumption reduction of pure electric vehicles. The conclusions are as follows:

(1) From the three aspects of speed, acceleration and driving state, the extraction parameters of the running characteristics of pure electric vehicles are proposed. The parameter contains 19 indexes, and the calculation method of each index is proposed.

(2) The driving cycle with four operating states of acceleration, deceleration, idling and constant speed (10, $20,30,40$, and 50) was proposed, and the proportion and slope of each state were scientifically determined according to the results of data analysis

Due to the limitations of the research, there are still some areas to be improved in the future research. Since the data collection is only for cities, the research in this paper does not cover the situation of intercity operation, which will be improved in further research.

\section{Acknowledgments}

This work was supported in part by the Special funds for basic research operations of Research Institute of Highway Ministry of Transport, grant number (20189050, 2020-9063, 2020-9064).

\section{References}

1. Millo F, Cubito C, Rolando L, et al. Design and development of a hybrid light commercial vehicle [J]. Energy, 2017, 136(10): 90-99.

2. Amirjamshidi G, Roorda MJ. Development of simulated driving cycles for light, medium, and heavy duty trucks: Case of the Toronto Waterfront Area [J]. Transportation research part D, 2015, 34(1): 255-266.

3. Fotouhi A, Montazeri-Gh M. Tehran driving cycle development using the K-means clustering method [J]. Scientia Iranica, 2013, 20(2): 286-293.

4. Liu Z, Ivanco A, Filipi Z. Naturalistic drive cycle synthesis for pickup trucks. Journal of safety research, 2015; 54: 109. 29-115 
5. Liu D, Zou Y, Liu T. Real-Time Markov Chain Driver Model for Tracked Vehicles [J]. Ifac Papers online, 2015, 48(15):361-367. 